\title{
Notas sobre educación media superior desde la hermenéutica analógica
}

\author{
José Ángel del Moral Palacio
}

\section{Resumen}

$\mathrm{E}$

ste texto presenta una reflexión acerca de los lineamientos para una praxis docente en la educación media superior. Se pretende partir de un fundamento teórico diverso, aunque complementario, al de las exposiciones que usualmente se hacen sobre el tema. La reflexión se refiere a la educación media superior de manera general, aunque el autor tenga por referente inmediato su propia praxis docente en el marco del programa de bachillerato perteneciente a la Universidad Autónoma de Aguascalientes (UAA). Se utiliza, como horizonte teórico y metodológico, la perspectiva filosófica de la hermenéutica analógica (HA), a través de la cual se hace una interpretación de aspectos fundamentales del sentido y praxis educativa en este tipo de educación.

Palabras clave: educación media superior, praxis docente, hermenéutica analógica.

\section{Introducción}

\section{La educación media superior}

De acuerdo con el artículo 37 de la Ley General de Educación, en México la educación se estructura en tres tipos: básico (preescolar, primaria y secundaria), medio-superior (bachillerato y equivalentes, y educación profesional que no requiere bachillerato) y superior (licenciatura, especialidad, maestría, doctorado, opciones terminales previas a la conclusión de la licenciatura y educación normal). En dicho marco, la educación media superior (EMS) se ubica en el centro y tiene una importancia decisiva por el hecho de atender a un grupo poblacional -jóvenes entre 15 y 19 años de edad- que enfrenta cambios y retos relevantes desde una perspectiva antropológica, psicológica y cultural (económica, laboral, social, etc.), que significan dejar atrás la infancia y sentar las bases para consolidar su desarrollo personal y humano, a fin de integrarse en la sociedad de manera plena y responsable. Es por ello que la EMS es una prioridad social: no sólo se trata de preparar a los jóvenes con competencias para estudios superiores, sino para la vida (UAA, 2018: 8).

La Secretaría de Educación Pública (SEP) la ha abordado mediante la Reforma Integral de la Educación Media Superior (RIEMS). En la Universidad Autónoma de
Aguascalientes (UAA), de forma similar a otras instituciones educativas, se hace mediante su actual Bachillerato Currículo 2018, y pretende, así, responder a las orientaciones de su propio Modelo Educativo Institucional (MEI) y a la RIEMS, incluido el Nuevo Modelo Educativo para la Educación Obligatoria (2017) (UAA, 2018: 6 y 59).

La justificación de estas reformas y programas se basa en propuestas de corte constructivista, así como en ciertas políticas educativas, como la del "Informe de la Comisión Internacional sobre la Educación para el Siglo XXI" de la UNESCO, en el que se proponen cuatro pilares para la educación -aprender a conocer, a hacer, a convivir y a ser-, de mano del modelo de educación por competencias (UNESCO, 1996: 96-108; UAA, 2018: 32). Por ello, el propósito de este texto es presentar unas breves notas sobre la EMS desde una perspectiva diversa y, a la vez, complementaria, de índole filosófica, mediante la hermenéutica analógica (HA).

\section{La hermenéutica analógica}

La hermenéutica es la disciplina encargada de la interpretación de los textos; no sólo de mensajes verbales, sino también de manifestaciones artísticas o religiosas, acontecimientos, acciones e interacciones (educar, por ejemplo). 
Interviene donde no hay un solo sentido, sino varios; su propósito es comprender, por lo que su medio principal es la contextualización. Interpretar consiste en poner un texto en su contexto, evitando así la mala comprensión (Beuchot, 2005: 15-17). En el presente caso, por ejemplo, se trata de comprender la praxis concreta de la EMS, ubicándola en su contexto, considerando que hay múltiples perspectivas implicadas (antropológica, psicológica, económica, social, pedagógica, etcétera).

$\ll$ Es por ello que la Ems es una prioridad social: no sólo se trata de preparar a los jóvenes con competencias para estudios superiores, sino para la vida.》〉

La HA, por su parte, es un método, un modo de pensamiento en el que se trata de salvaguardar las diferencias en el margen de cierta unidad (Beuchot, 1996: 61). Es una forma de pensamiento intermedio entre una hermenéutica unívoca, de corte cientificista (de quienes afirman que sólo una interpretación es válida); y la equívoca, de corte relativista (de quienes sostienen que toda interpretación es válida). Si hay diversidad de interpretaciones, la HA no renuncia a cierta uniformidad, a convenir en algo estable y reconocible, de forma que sea posible un conocimiento razonable (Beuchot, 2005: 37-38; Del Moral, 2013: 136-137).

\section{Hermenéutica analógica y educación media superior}

En el caso de la EMS, se puede caer en el extremo univocista de pretender que la única interpretación válida es seguir los programas de manera fundamentalista, al pie de la letra, sin considerar circunstancias concretas, o que su única finalidad sea la incorporación del estudiante a la educación superior o al mercado laboral. Se puede caer también en el equivocismo, al considerar un programa como una mera guía y aplicar su contenido de manera personal y subjetiva, a la sazón de las circunstancias y los estados de ánimo temporales. Por ello, es importante una hermenéutica analógica, que abra el campo de interpretaciones válidas, pero que también le ponga límites (Beuchot, 2005: 11; Del Moral, 2013: 136-137).

Hay que ubicar, ante todo, a la EMS en el contexto de la educación en general, en cuyo marco se observa la estructura hermenéutica del proceso educativo: en la interacción docente-alumno hay una interpretación de los alumnos hecha por el docente y una interpretación del docente hecha por los alumnos (Beuchot, 2009: 39-41). En este proceso, la HA procura evitar los extremos de posturas univocistas (autoritarias y de disciplina férrea) y equivocistas (un permitir hacer excesivo que deja casi todo en manos de los alumnos), pues busca un equilibrio armónico intermedio que permita la apertura y flexibilidad sin perder el rigor necesario del aprendizaje (Beuchot, 2014: 155). Pretende que la relación docente-alumno -sin que caiga en imposiciones ni rigidez (de forma mecánica o sin sentido)- sea lo suficientemente seria y objetiva, y-sin que se dé con relativismos o de forma meramente intuitiva- sea abierta y fluida (Beuchot, 2009: 42; Del Moral, 2014: 18-19); pues tanto el extremo univocista como el equivocista privan al ser humano de una auténtica educación, concebida como formación de hábitos, virtudes (Beuchot, 2018: 152-153; Del Moral, 2014: 34-36) o, como es costumbre denominarlas actualmente, de actitudes.

Sería erróneo identificar a la educación tradicional con el univocismo. En efecto, se habla mucho de "nuevos" modelos pedagógicos (conductivismo, constructivismo, etc.), que no conciben la educación como transmisión de contenidos, sino como formación, a diferencia del "tradicional", que habría que superar; es una afirmación correcta sólo parcialmente. La educación no es sólo transferencia de contenidos; es formación que implica necesariamente cierta transmisión de contenidos (matemáticas, español, historia, etc.), como señala la Ley General de Educación: "La educación es medio fundamental para adquirir, transmitir y acrecentar la cultura; es proceso permanente que contribuye al desarrollo del individuo y a la transformación de la sociedad, y es factor determinante para la adquisición de conocimientos y para formar a mujeres y a hombres, de manera que tengan sentido de solidaridad social" (art. 2). Si, por ejemplo, en la UAA se concibe -acertadamente- la educación como un proceso intencional y sistemático, cuya finalidad es la formación integral de la persona (UAA, 2007: 6), dicha formación incluye la transmisión de contenidos. La dificultad estriba en la forma de la transmisión: unívoca (impositiva, acrítica), equívoca (arbitraria) o análoga (significativa, pertinente). Es imprescindible, al lado de la transmisión de contenidos, la formación de hábitos: aprender a conocer, a hacer, a convivir y a ser.

Por otra parte, el lugar que ocupa la EMs entre la educación básica y la superior habla de su función como puente que proporcione adecuadamente el tránsito entre dichos tipos, y en el que se formen y consoliden los hábitos. Tan sólo por considerar la dimensión antropopsicológica, es en dicha estructura en la que se debe situar la 
problemática de la (in)madurez en los adolescentes. No se trata de considerar la adolescencia como una situación in se, sino como una "etapa hacia la madurez", que apunta a la inserción en la vida social y laboral de los ahora jóvenes como ciudadanos adultos responsables y solidarios. Es importante considerarla así para no caer en los extremos de demasiada condescendencia o rigor hacia los jóvenes, o en permitirles cierto infantilismo o tratarlos como adultos. Lograr este punto intermedio, análogo, es una gran oportunidad para la EMS, de la cual depende la formación de ciudadanos responsables y solidarios -reto vinculado con la adecuada relación entre derechos y deberes del joven, así como con su relación con las figuras de autoridad-, pues se forma para la vida, el trabajo, conformar las futuras familias, la sociedad, etc. No hay que considerar la adolescencia en sí, sino como una etapa hacia la vida adulta y responsable en el marco de una formación para la libertad con compromiso, sin caer en la infantilización, manteniéndolos en una etapa de irresponsabilidad; ni de considerarlos ya como adultos, porque no lo son.

Respecto a los hábitos, gran parte de la tradición considera que educar consiste en la formación de virtudes, pues la mera información, privada de hábitos para asimilarla, comprenderla y aplicarla de manera integral y humana, es "ciega", como materia sin forma. Pero no hay métodos o reglas para enseñar la virtud; ésta se aprende a partir de ciertos principios generales, pero, sobre todo, con modelos y práctica. La virtud modelo de todas las demás que dirige el actuar humano es la prudencia (Aristóteles, 1988, Ética Nicomáquea, vI, 4-5; 1140-32), la virtud del bien actuar concreto que enseña cómo comportarse en

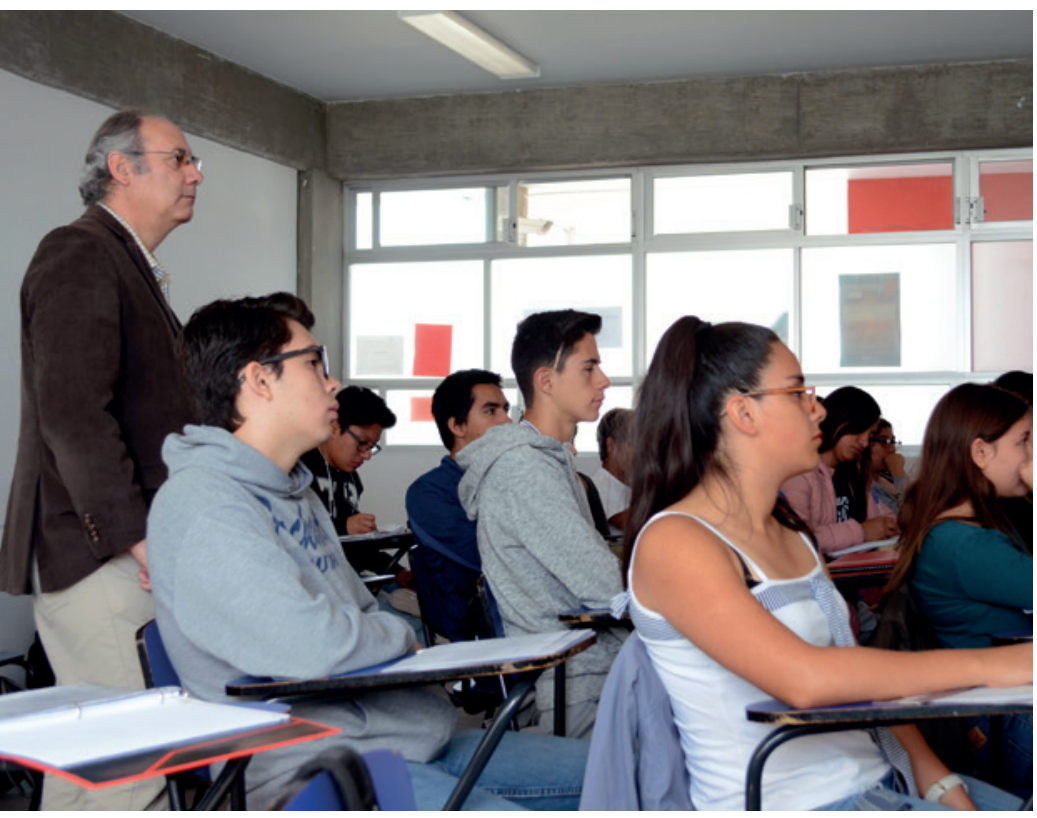

el caso concreto, en el momento preciso; es el saber de los medios, no sólo del medio proporcional en que consiste la virtud, sino de los medios respecto a los fines. La prudencia hace elegir los medios adecuados en orden al fin, o hace aplicar adecuadamente normas generales en casos concretos, considerando las circunstancias. Consta de dos partes: deliberación (sopesar los pros y los contra de los medios para la acción) y decisión (el juicio: determinar qué medio es el más oportuno para alcanzar el fin); por eso, entre los griegos, la educación del ciudadano residía en el aprendizaje de la deliberación: era ciudadano el que era capaz de deliberar, de ahí que el objetivo de la educación sea formar el juicio, tanto teórico como práctico; formar asimismo el criterio, esto es, enseñar a deliberar (Beuchot, 2018: 148-150).

《 La HA procura evitar los extremos de posturas univocistas (autoritarias y de disciplina férrea) y equivocistas (un permitir hacer excesivo que deja casi todo en manos de los alumnos), pues busca un equilibrio armónico intermedio.》》

Una siguiente anotación se refiere a la insistencia en la formación integral y pertinente de los programas actuales de EMs, lo que va muy de la mano con la proporción, el equilibrio y la analogía. Ahora bien, esto conlleva, además, la justa y prudente aplicación de programas, normativas, reglamentos y metodologías, evitando seguirlos literalmente (univocismo) o de manera relativa, espontánea o voluntarista (equivocismo), teniendo siempre en cuenta a las personas y situaciones concretas. El fin de la educación es el desarrollo integral de la persona, de seres humanos concretos; las normas, reglamentos y métodos son medios. No se relativizan haciéndolos menos, sino que se contextualizan poniéndolos en su lugar: no son el fin; el fin son las personas. "El mal -dice Tomás de Aquino- consiste en convertir los medios en fin o el fin en medios" (Super Sent., lib. 1, d. 1, q. 4, a. 2, expos.). El documento citado de la UNESCO subraya este aspecto al hablar del cuarto pilar: "aprender a ser" (1996: 106-107), referido al desarrollo integral de la persona: ser de manera plena, integral, lo que conlleva la capacidad de pensamiento autónomo y crítico, así como de elaborar juicios propios para determinar por sí mismos qué hacer en las diferentes circunstancias de la vida.

$Y$ añade: no se trata tanto de que los jóvenes aprendan a adaptarse a una sociedad (tecnológica u otra cual- 
quiera), sino más bien dotar a cada quien de fuerzas y puntos intelectuales permanentes de referencia que le permitan comprender el mundo que le rodea y comportarse como un elemento responsable y justo. La función esencial de la educación es, en ese sentido, conferir a los seres humanos libertad de pensamiento, de juicio, de sentimientos y de imaginación, con el objetivo de que sus capacidades alcancen la plenitud y sean artífices, en la medida de lo posible, de su destino. El equilibrio analógico se hace ahí presente: no se trata de presentar contenidos no significativos, pero tampoco de formar sólo para este mundo, más bien de formar para habitar humanamente el mundo que le rodea, cualquiera que éste sea.

Un elemento esencial en este proceso es la estructura dialógica de la enseñanza: es difícil que alguien, por sí mismo, encuentre sus deficiencias, sus puntos ciegos; se necesita el diálogo con el otro. Mediante el diálogo, las personas se ayudan y enriquecen mutuamente. Es imprescindible la interpelación por el otro, lo que convierte al proceso educativo impositivo en diálogo (Beuchot, 2009: 48-49). Pero "dialogar" significa que ambos interlocutores hablan y se escuchan; lo distinto al diálogo significa el monólogo (univocismo impositivo) o el caos relativista en que ambos hablan sin escucharse ni comprenderse (equivocista). De ahí la importancia de la educación en, por y para el diálogo (Del Moral, 2014: 12). De la misma manera, debe haber comprensión y juicio: no se puede dialogar con quien no se comprende; no se puede evitar emitir juicios acerca de lo que se comprende (Beuchot, 2009: 31). Por todo ello, hay que prestar más atención a la formación para el diálogo, para la relación, el respeto y el reconocimiento del otro; en otros términos, a la formación para escuchar, comprender, discernir y emitir juicios; formación para abrirse al otro, dejarse interpelar y vivir en solidaridad (Del Moral, 2014: 35-36).

En fin, desde el horizonte de la racionalidad, la formación integral se manifiesta como equilibrio entre la dimensión argumentativa y epistémica, y la dimensión emotivo-sentimental y axiológica. Adela Cortina dice que la razón integral es razón "cordial", pues lo más importante de la vida humana no se conoce sólo mediante la argumentación, sino también a través del "corazón" (Cortina, 2007: 191), que caracteriza así:

La competencia comunicativa y la capacidad de entablar un diálogo presupongan inevitablemente [la capacidad de estimar los valores, la capacidad de sentir y la capacidad de formarse un juicio justo a través de la adquisición de las virtudes. Este conjunto de capacidades compone el corazón de las personas], que es el lugar del afecto, pero también de la inteligencia, el espíritu, el talento, incluso el estómago para acometer grandes empresas, $\mathrm{y}$ es el que se reconocen mutuamente quienes realizan acciones comunicativas, si es que su ingreso en la comunicación tiene un sentido (Cortina, 2010: 16).

Mediante la estimación de valores, sentir, formarse un juicio y adquirir virtudes se comprende y habita en el mundo "cordialmente" de manera integral, evitando los extremos del racionalismo argumentativo y del irracionalismo emotivista (Del Moral, 2018: 37).

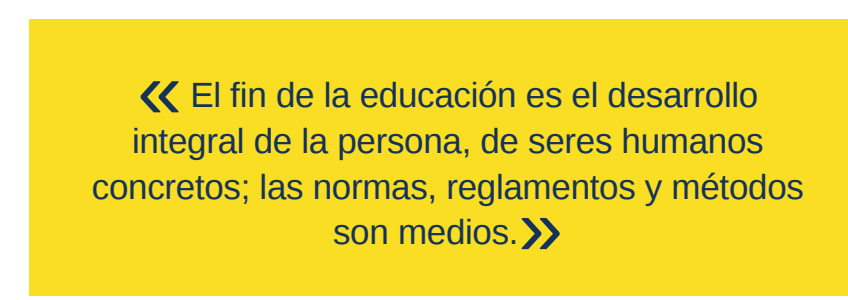

\section{Conclusión}

La hermenéutica analógica nos presenta pautas que permiten interpretar de forma general la dinámica del proceso de enseñanza-aprendizaje en educación media superior desde la filosofía, lo que constituye una forma de comprenderlo de manera distinta y, a la vez, complementaria a las perspectivas que se utilizan usualmente en el ámbito de las instituciones educativas y de las políticas públicas. Esto significa una riqueza para profundizar en la propia praxis docente y en el sentido y formas de la educación. Por otra parte, su aplicación a casos concretos en la implementación de programas educativos va más allá de la mera comprensión, pues supone la consideración de las circunstancias específicas en cada proyecto en que se inscribe.

Como puede observarse, lo dicho acerca de la educación media superior es susceptible de aplicación, análoga, a su vez, a los distintos tipos y niveles educativos. Esto significa que las anotaciones hechas muestran cómo las posibilidades de una interpretación analógica de la praxis educativa y pedagógica son mucho más amplias de los límites planteados en el presente texto. Es muy largo el camino a recorrer y numerosos los senderos por donde transitar desde este horizonte. Sólo hacía falta iniciar el trayecto. 

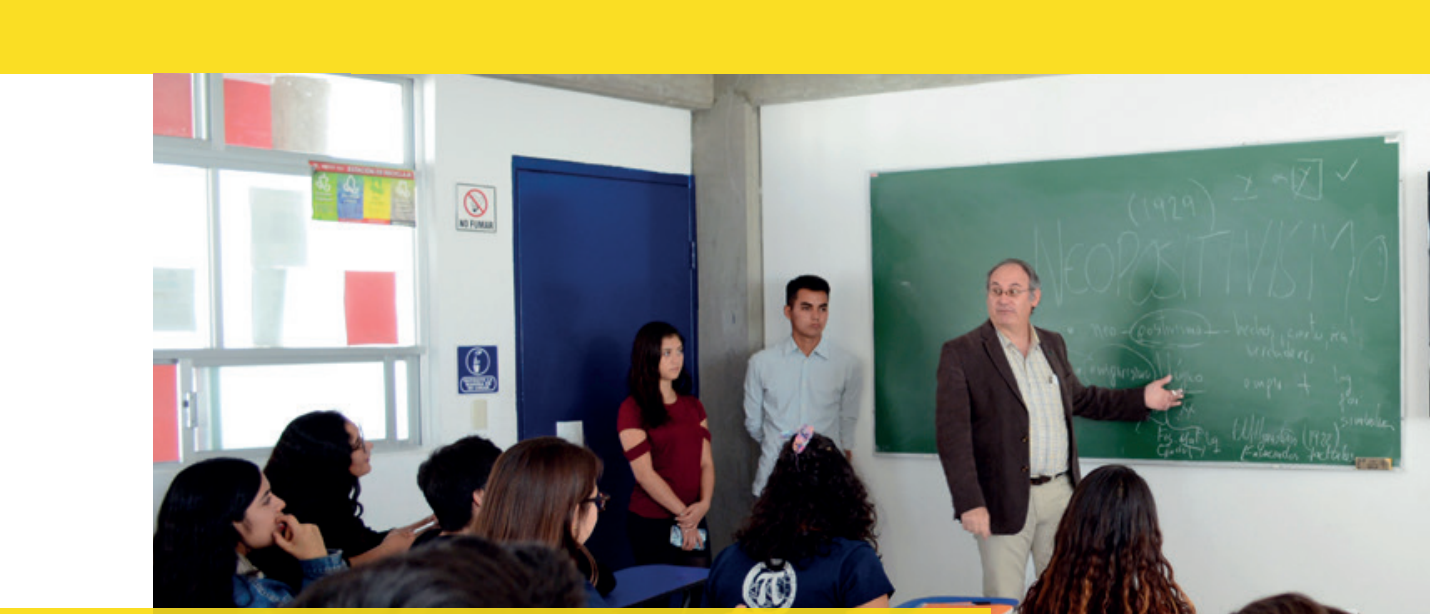

José Ángel del Moral Palacio

Hay que prestar más atención a la formación para el diálogo, para la relación, el respeto y el reconocimiento del otro; en otros términos, a la formación para escuchar, comprender, discernir y emitir juicios; formación para abrirse al otro, dejarse interpelar y vivir en solidaridad. \)
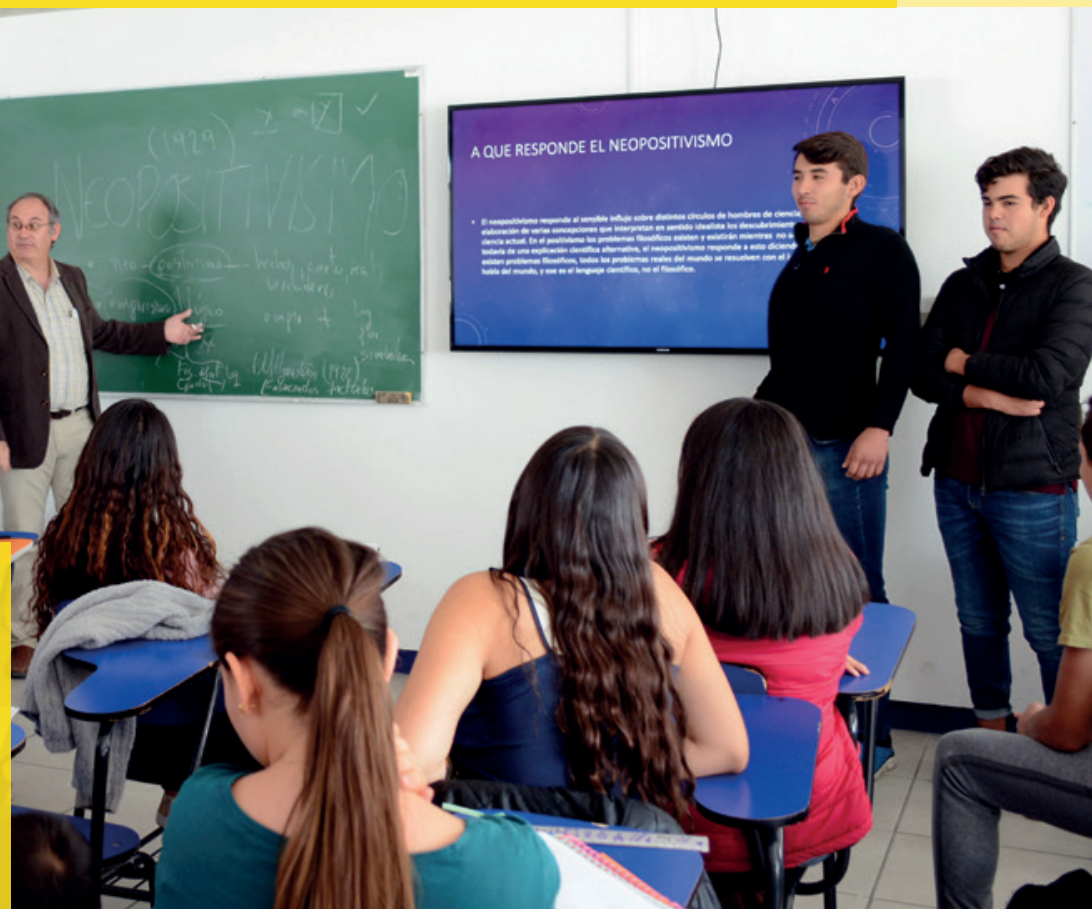
$a$
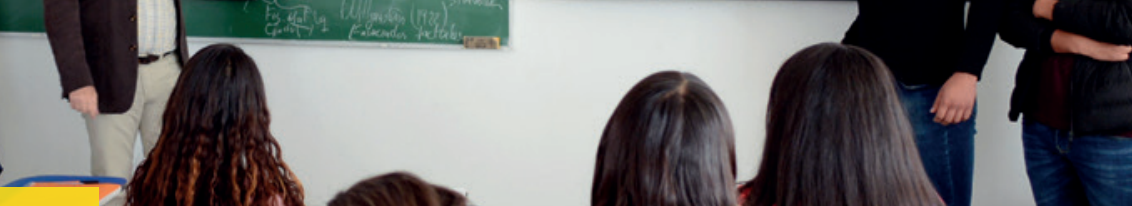